\title{
Optimization of Training Signal Transmission for Estimating MIMO Channel under Antenna Mutual Coupling Conditions
}

\author{
Xia Liu and Marek E. Bialkowski \\ School of ITEE, The University of Queensland, Brisbane QLD 4072, Australia \\ Correspondence should be addressed to Xia Liu, xialiu@itee.uq.edu.au \\ Received 2 December 2009; Revised 26 February 2010; Accepted 17 March 2010 \\ Academic Editor: Hon Tat Hui \\ Copyright ( $) 2010$ X. Liu and M. E. Bialkowski. This is an open access article distributed under the Creative Commons Attribution \\ License, which permits unrestricted use, distribution, and reproduction in any medium, provided the original work is properly \\ cited. \\ This paper reports investigations on the effect of antenna mutual coupling on performance of training-based Multiple-Input \\ Multiple-Output (MIMO) channel estimation. The influence of mutual coupling is assessed for two training-based channel \\ estimation methods, Scaled Least Square (SLS) and Minimum Mean Square Error (MMSE). It is shown that the accuracy of \\ MIMO channel estimation is governed by the sum of eigenvalues of channel correlation matrix which in turn is influenced by \\ the mutual coupling in transmitting and receiving array antennas. A water-filling-based procedure is proposed to optimize the \\ training signal transmission to minimize the MIMO channel estimation errors.
}

\section{Introduction}

In recent years, there has been a growing interest in multiple-input multiple-output (MIMO) wireless communication systems as they can significantly increase data throughput (capacity) without the need for extra operational frequency bandwidth. In order to explore the advantages of MIMO technique, precise channel state information (CSI) is required at the receiver. The reason is that without CSI, decoding of the received signal is impossible [1-5]. In turn, an inaccurate CSI leads to an increased bit error rate (BER) that translates into a degraded capacity of the system [6-8].

Obtaining accurate CSI can be accomplished using suitable channel estimation methods. The methods based on the use of training sequences, known as the trainingbased channel estimation methods, are the most popular. In $[9,10]$, several training-based methods including the least square (LS) method, the scaled least square (SLS) method and the minimum mean square error (MMSE) method have been investigated. It has been shown that the accuracy of these training-based estimation methods is influenced by the transmit signal power to noise ratio (SPNR) in the training mode, and the number of antenna elements at the transmitter and receiver. In particular, it has been pointed out that when the transmitted SPNR and the number of antenna elements are fixed, the SLS and MMSE methods offer better performance than the LS method. This can be explained by the fact that the SLS and MMSE methods utilize the channel correlation in the estimator cost function while the LS estimator does not take into account the channel properties.

It is worthwhile to note that the channel properties are governed by a signal propagation environment, which in turn affects the spatial correlation (SC) observed at the input/output ports of the MIMO system. The spatial correlation is dependent on an antenna configuration and a distribution of scattering objects that are present in the path between transmitter and receiver. In particular it is influenced by the finite antenna spacing in array antennas. This finite antenna spacing is also responsible for mutual coupling which adversely affects signal power transmission and reception. The mutual coupling effect is especially pronounced in tightly spaced arrays. Because there is a considerable demand for compact size Mobile Station (MS) terminals, the effect of mutual coupling cannot be neglected and thus has to be taken into account while assessing the MIMO link performance. The problem of mutual coupling in MIMO systems for the case of peer-to-peer communication has been addressed via simulations and measurements 
in [10-18]. It has been shown that the mutual coupling may improve the channel capacity when the spacing of antenna elements in the array is between $0.2 \lambda$ to $0.4 \lambda$ (where $\lambda$ is the carrier wavelength) [15-18] .

In this paper, the focus is on the effect of mutual coupling on MIMO channel estimation. The problem of mutual coupling and its effect on the MMSE method of channel estimation have been investigated in $[19,20]$. The presented results have been limited to simulations providing general trends without any further mathematical insight.

In this paper, we present a mathematical analysis explaining the mutual coupling effects on the SLS and MMSE methods for channel estimation. It is shown that for a fixed transmit SPNR and for a given number of transceiver antenna elements the accuracy of SLS and MMSE methods is determined by the sum of eigenvalues of channel correlation matrix characterizing the signal propagation conditions. The presence of mutual coupling in array antennas causes variations of the sum of these eigenvalues, which in turn affects the accuracy of estimation. In order to reduce the channel estimation errors, a water-filling based optimization method is proposed to find an optimal training matrix minimizing the channel estimation errors when the MIMO system operates under the condition in which the mutual coupling cannot be neglected.

The rest of the paper is organized as follows. In Section 2, a MIMO system model is introduced. Also, LS, SLS, and MMSE channel estimation methods are described followed by an analysis of channel estimation accuracy. Section 3 describes the method for modeling mutual coupling effects. Section 4 presents the optimization of a training signal transmission when the MIMO system operates under mutual coupling conditions. Section 5 describes computer simulation results. Section 6 concludes the paper.

\section{System Description and Training-Based MIMO Channel Estimation}

In this paper, a narrow band block fading MIMO channel is assumed. The number of transmitting and receiving antennas is denoted as $M_{t}$ and $M_{r}$, respectively. The channel is described by the $M_{r} \times M_{t}$ complex matrix $H$ with entries $h_{i j}$ representing the response between the $i$ th receiving antenna and the $j$ th transmitting antenna.

2.1. Training-Based MIMO Channel Estimation. For the training-based channel estimation method, the relationship between the received signal and the training sequence is given by

$$
Y=H P+V
$$

where $P$ represents the $M_{t} \times L$ complex training matrix and $L$ is the length of the training sequence.

The goal is to estimate the complex channel matrix $H$ from the knowledge of $Y$ and $P$. The transmitted power over $L$ time slots in the training mode is constrained by the following expression

$$
\|P\|_{F}^{2}=P
$$

where $P$ is a given constant representing the total power and $\|\cdot\|_{F}^{2}$ stands for the Frobenius norm.

The training matrices are assumed to be orthogonal [9, $10]$ and the transmitted signal power to noise ratio (SPNR) in the training mode is set to $\rho$ equal to $P / \sigma_{n}^{2}$.

Using the LS method, the estimated channel matrix can be written as [19]

$$
\hat{H}_{\mathrm{LS}}=Y P^{\dagger}
$$

where $\{\cdot\}^{\dagger}$ stands for the pseudo-inverse operation and $P^{\dagger}=$ $P^{H}\left(P P^{H}\right)^{-1}$. The mean square error (MSE) of the estimated channel matrix in the LS method is given as

$$
\operatorname{MSE}_{\mathrm{LS}}=E\left\{\left\|H-\hat{H}_{\mathrm{LS}}\right\|_{F}^{2}\right\} \quad \text { subject to }\|P\|_{F}^{2}=P \text {. }
$$

According to $[9,10]$, the optimal training sequence satisfying (4) is given as

$$
P P^{H}=\frac{P}{M_{t}} I .
$$

The minimum value of MSE for the LS method is

$$
\mathrm{MSE}_{\mathrm{LS}}=\frac{M_{t}^{2} M_{r}}{\rho} .
$$

From (6) one can see that the optimal performance of the LS estimator is influenced by the number of antenna elements at the transmitter and the receiver. However, the channel matrix has no effect on the minimum value of MSE.

The SLS method further reduces the estimation error that is obtained in the LS method. The improvement is given by a scaling factor $\gamma$ which uses the channel estimator as given by

$$
\hat{H}_{\mathrm{SLS}}=\gamma \hat{H}_{\mathrm{LS}}
$$

The resulting estimation error is given by (8)

$$
\mathrm{MSE}_{\mathrm{SLS}}=E\left\{\left\|H-\gamma \hat{H}_{\mathrm{LS}}\right\|_{F}^{2}\right\} \quad \text { subject to }\|P\|_{F}^{2}=P \text {. }
$$

Expression (8) can be rewritten as (9)

$$
\begin{aligned}
\operatorname{MSE}_{\mathrm{SLS}}= & E\left\{\operatorname{tr}\left\{\left(H-\gamma \hat{H}_{\mathrm{LS}}\right)^{H}\left(H-\gamma \hat{H}_{\mathrm{LS}}\right)\right\}\right\} \\
= & E\left\{(1-\gamma)^{2} \operatorname{tr}\left\{R_{H}\right\}+\gamma^{2} \mathrm{MSE}_{\mathrm{LS}}\right\} \\
= & E\left\{\left(\mathrm{MSE}_{\mathrm{LS}}+\operatorname{tr}\left\{R_{H}\right\}\right)\left(\gamma-\frac{\operatorname{tr}\left\{R_{H}\right\}}{\mathrm{MSE}_{\mathrm{LS}}+\operatorname{tr}\left\{R_{H}\right\}}\right)^{2}\right. \\
& \left.+\frac{\mathrm{MSE}_{\mathrm{LS}} \operatorname{tr}\left\{R_{H}\right\}}{\mathrm{MSE}_{\mathrm{LS}}+\operatorname{tr}\left\{R_{H}\right\}}\right\},
\end{aligned}
$$


in which $R_{H}$ is the channel correlation matrix defined as $R_{H}=\left\{H^{H} H\right\}$.

From (9), it is apparent that in order to minimize the MSE of SLS, an optimal value of $\gamma$ as given by (10) has to be chosen

$$
\gamma=\frac{\operatorname{tr}\left\{R_{H}\right\}}{\operatorname{MSE}_{\mathrm{LS}}+\operatorname{tr}\left\{R_{H}\right\}} .
$$

The minimized value of MSE can be written as $[9,10]$

$$
\mathrm{MSE}_{\mathrm{SLS}}=E\left\{\frac{\mathrm{MSE}_{\mathrm{LS}} \operatorname{tr}\left\{R_{H}\right\}}{\mathrm{MSE}_{\mathrm{LS}}+\operatorname{tr}\left\{R_{H}\right\}}\right\}<\mathrm{MSE}_{\mathrm{LS}} .
$$

By substituting (6) into (11), the minimum value of MSE is expressed as,

$$
\mathrm{MSE}_{S L S}=E\left\{\frac{1}{1 / \operatorname{tr}\left\{R_{H}\right\}+\rho / M_{t}^{2} M_{r}}\right\} .
$$

The estimated channel matrix as given by SLS method can be obtained using (7) and is given as

$$
\begin{aligned}
\hat{H}_{\mathrm{SLS}} & =\gamma \hat{H}_{\mathrm{LS}} \\
& =\left\{\frac{\operatorname{tr}\left\{R_{H}\right\}}{\mathrm{MSE}_{\mathrm{LS}}+\operatorname{tr}\left\{R_{H}\right\}} Y_{p} P^{\dagger}\right\} .
\end{aligned}
$$

In practice, $R_{H}$ can be obtained using the channel matrix estimated by the LS method. Therefore

$$
\operatorname{tr}\left\{\hat{R}_{H}\right\}=\operatorname{tr}\left\{\hat{H}_{\mathrm{LS}}^{H} \hat{H}_{\mathrm{LS}}\right\} .
$$

In the MMSE method, the estimated channel matrix is given as [21],

$$
\hat{H}_{\mathrm{MMSE}}=Y \psi .
$$

The objective is to find the optimal $\psi$ to minimize the MSE. This task can be expressed as

$$
\begin{aligned}
\psi^{\mathrm{opt}} & =\arg \min \left\|H-\hat{H}_{\mathrm{MMSE}}\right\|_{F}^{2} \\
& =\underset{\psi}{\arg \min }\|H-Y \psi\|_{F}^{2} .
\end{aligned}
$$

The mean-square error (MSE) for the MMSE method is given as,

$$
\begin{aligned}
\operatorname{MSE}_{\text {MMSE }}= & E\left\{\|H-Y \psi\|_{F}^{2}\right\}, \\
= & E\left\{\operatorname{tr}\left\{R_{H}\right\}-\operatorname{tr}\left\{R_{H} P \psi\right\}-\operatorname{tr}\left\{\psi^{H} P^{H} R_{H}\right\}\right. \\
& \left.+\operatorname{tr}\left\{\psi^{H}\left(P^{H} R_{H} P+\sigma_{n}^{2} M_{r} I\right) \psi\right\}\right\} .
\end{aligned}
$$

The optimal $\psi$ minimizing MSE satisfies (18),

$$
\frac{\partial \mathrm{MSE}_{\mathrm{MMSE}}}{\partial \psi}=0 .
$$

Thus the optimal $\psi$ can be represented by

$$
\psi^{\mathrm{opt}}=\left(P^{H} R_{H} P+\sigma_{n}^{2} M_{r} I\right)^{-1} P^{H} R_{H} .
$$

The estimated channel matrix can be derived as,

$$
\hat{H}_{\mathrm{MMSE}}=Y \psi^{\mathrm{opt}}=Y\left(P^{H} R_{H} P+\sigma_{n}^{2} M_{r} I\right)^{-1} P^{H} R_{H} .
$$

By defining the channel estimation error as

$$
e=H-\hat{H}_{\mathrm{MMSE}},
$$

the MSE for the MMSE method can be expressed as

$$
\begin{aligned}
\operatorname{MSE}_{\mathrm{MMSE}} & =E\left\{\operatorname{tr}\left\{R_{E}\right\}\right\} \\
& =E\left\{\operatorname{tr}\left\{E\left\{e e^{H}\right\}\right\}\right\} \\
& =E\left\{\operatorname{tr}\left\{\left(R_{H}^{-1}+\frac{1}{\sigma_{n}^{2} M_{r}} P P^{H}\right)^{-1}\right\}\right\} \\
& =E\left\{\operatorname{tr}\left\{\left(\Lambda^{-1}+\sigma_{n}^{-2} M_{r}^{-1} Q^{H} P P^{H} Q\right)^{-1}\right\}\right\} .
\end{aligned}
$$

In (22), $Q$ is a unitary eigenvector matrix of $R_{H}$ and $\Lambda$ is a diagonal matrix with eigenvalues of $R_{H}$, which are given through the eigenvalue decomposition of $R_{H}$ as

$$
R_{H}=H^{H} H=Q \Lambda Q^{H} .
$$

Here, to construct the channel matrix $H$, the Kronecker channel model is postulated $[22,23]$. In this model, the transmitter and receiver correlation matrices are assumed to be separable and the channel matrix is represented as:

$$
H=R_{R}^{1 / 2} G_{H} R_{T}^{1 / 2},
$$

where $G_{H}$ is the matrix including identical independent distributed (i.i.d) Gaussian entries with a zero mean and a unit variance, and $R_{R}$ and $R_{T}$ are the spatial correlation matrices at the receiver and transmitter, respectively.

The superiority of SLS and MMSE methods over LS method is due to the fact that SLS and MMSE methods utilize information of the channel correlation $R_{H}$. Here, it is assumed that the $R_{H}$ is perfectly obtained before channel estimation.

In further considerations, it is assumed that the transmitting and receiving array antennas are formed by vertically polarized wire dipole antennas which are surrounded by scattering objects. Assuming that these scattering objects are uniformly distributed within circles surrounding the array antennas, the spatial correlation matrix elements can be obtained using the Clark's model as given by.

$$
\rho_{i, j}^{R(T)}=J_{0}\left(\kappa d_{i, j}\right) .
$$

where $J_{0}$ stands for the zero-order Bessel function, $\kappa$ is a wave number and $d_{i j}$ is the distance between elements $i$ and $j$ of 
the uniform array antenna. The correlation matrices $R_{T}$ and $R_{R}$ can be generated using (25) as

$$
R_{R(T)}=\left[\begin{array}{ccc}
\rho_{1,1}^{R(T)} & \cdots & \rho_{1, M_{r(t)}}^{R(T)} \\
\vdots & \ddots & \vdots \\
\rho_{M_{r(t)}, j}^{R(T)} & \cdots & \rho_{M_{r(t)}, M_{r(t)}}^{R(T)}
\end{array}\right]
$$

Having determined $R_{T}$ and $R_{R}$, the channel matrix can be calculated using (24).

2.2. Estimation Accuracy Analysis. By taking into account expression (23), the minimized MSE of the SLS method (12) can be rewritten as

$$
\begin{aligned}
\operatorname{MSE}_{S L S} & =E\left\{\left[\left(\operatorname{tr}\left\{R_{H}\right\}\right)^{-1}+\frac{\rho}{M_{t}^{2} M_{r}}\right]^{-1}\right\} \\
& =E\left\{\left[(\operatorname{tr}\{\Lambda\})^{-1}+\frac{\rho}{M_{t}^{2} M_{r}}\right]^{-1}\right\} \\
& =E\left\{\left[\left(\sum_{i}^{n} \lambda_{i}\right)^{-1}+\frac{\rho}{M_{t}^{2} M_{r}}\right]^{-1}\right\},
\end{aligned}
$$

where $n=\min \left(M_{r}, M_{t}\right)$ and $\lambda_{i}$ is the $i$ th eigenvalue of the channel correlation matrix $R_{H} \cdot \rho$ is the transmit SPNR and equal to $P / \sigma_{n}^{2} . P$ is the transmitted training sequence total power. If power $P$ and the number of transmit and receive antennas are fixed then the following relationship holds

$$
\operatorname{MSE}_{\text {SLS }}=E\left\{\left[\left(\sum_{i}^{n} \lambda_{i}\right)^{-1}+\frac{\rho}{M_{t}^{2} M_{r}}\right]^{-1}\right\}<E\left\{\sum_{i}^{n} \lambda_{i}\right\} .
$$

It can be seen from (28) that $\mathrm{MSE}_{\mathrm{SLS}}$ is smaller than the sum of the eigenvalues of the channel correlation matrix $R_{H}$. Therefore, the sum of the eigenvalues of the channel correlation matrix $R_{H}$ is the upper bound of MSE in the SLS method.

As observed from expression (28), MSE decreases when the sum of eignvalues of $R_{H}$ decreases. The same expression shows that the MSE in the SLS method is influenced by the number of antenna elements at the transmitter and receiver. When the number of antenna elements on the two sides of communication link drops to one, the system becomes the conventional SISO system. In this case, the channel estimation using a fixed-length training sequence becomes most accurate. This confirms the expectation that it is easier to estimate the SISO channel which is characterized by a single transfer coefficient between single transmitting and receiving antennas than the MIMO channel which is described by a matrix of transfer coefficients between many antenna elements.

The derived expression also shows that when the number of transmit and receive antennas and the total transmitted power $P$ is fixed, MSE can be minimized by minimizing the sum of eigenvalues of $R_{H}$.
The expression (22) for the minimized value of MSE can be rewritten using the orthogonality properties of a training sequence $P$ and the unitary matrix $Q$, as shown by

$$
\begin{aligned}
& \text { MSE }_{\text {MMSE }} \\
& =E\left\{\operatorname{tr}\left\{\left(\Lambda^{-1}+\rho M_{r}^{-1} I\right)^{-1}\right\}\right\} \\
& =E\left\{\operatorname{tr}\left\{\left[\begin{array}{cccc}
\left(\lambda_{1}^{-1}+\rho M_{r}^{-1}\right)^{-1} & 0 & \cdots & 0 \\
0 & \left(\lambda_{2}^{-1}+\rho M_{r}^{-1}\right)^{-1} & \ddots & \vdots \\
\vdots & \ddots & \ddots & 0 \\
0 & \cdots & \left(\lambda_{n}^{-1}+\rho M_{r}^{-1}\right)^{-1}
\end{array}\right]\right\}\right\} \\
& =E\left\{\sum_{i}^{n}\left(\lambda_{i}^{-1}+\rho M_{r}^{-1}\right)^{-1}\right\} .
\end{aligned}
$$

Similar derivations for MSE apply for the MMSE method. By using the steps analogous to those used for the SLS method, the MSE upper bound for the MMSE method can be expressed as

$$
\operatorname{MSE}_{\mathrm{MMSE}}=E\left\{\sum_{i}^{n}\left(\lambda_{i}^{-1}+\rho M_{r}^{-1}\right)^{-1}<\sum_{i}^{n} \lambda_{i}\right\} .
$$

The expression (30) shows that similarly as in the SLS method, a smaller sum of eigenvalues of the channel correlation matrix $R_{H}$ leads to a smaller estimation error in the MMSE method. Similarly as in the SLS method, the MSE of MMSE is influenced by the number of transmitting and receiving antennas.

Through the above mathematical analysis, one can see that if the total transmitted power $P$ as well as the number of antenna elements at the transmitter and the receiver is fixed, the accuracy of the training-based MIMO channel estimation is governed by the sum of eigenvalues of the channel correlation matrix $R_{H}$. The smaller is the sum, the more accurate channel estimation is achieved.

Decreasing the sum of eigenvalues of channel correlation is equivalent to decreasing the effective degree of freedom (EDOF) of the MIMO channel [24]. This is caused by an increased spatial correlation [25]. Hence, it is apparent that an increased spatial correlation helps improving the channel estimation accuracy.

\section{Mutual Coupling Effects}

Mutual coupling in an array of collinear side-by-side wire dipoles can be modeled using the approach described in [26]. Assuming the array is formed by $M_{r t}$ wire dipoles, the mutual matrix can be calculated using the following relationship with the impedance matrix

$$
\mathbf{C}=\left(Z_{A}+Z_{T}\right)\left(Z+Z_{T} \mathbf{I}_{M_{r t}}\right)^{-1},
$$

where $Z_{A}$ is the element impedance in isolation, for example, when the wire dipole is $\lambda / 2$, its value is $Z_{A}=73+j 42.5[\Omega]$; $Z_{T}$ is impedance of the receiver at each element chosen as 
the complex conjugate of $Z_{A}$ to obtain the impedance match. The mutual impedance matrix $\mathbf{Z}$ is given by

$$
\mathbf{Z}=\left[\begin{array}{cccc}
Z_{A}+Z_{T} & Z_{12} & \cdots & Z_{1 M_{r t}} \\
Z_{21} & Z_{A}+Z_{T} & \cdots & Z_{2 M_{r t}} \\
\vdots & \vdots & \ddots & \vdots \\
Z_{M_{r t} 1} & Z_{M_{r t} 2} & \cdots & Z_{A}+Z_{T}
\end{array}\right]
$$

Note that this expression provides the circuit representation for the mutual coupling in array antennas. It is valid for antennas operating in a single mode. Wire dipoles fall into this category.

For a side-by-side array configuration of dipoles having length 1 equal to $0.5 \lambda$, the expressions for $\left\{Z_{m n}\right\}$ can be adapted from $[18,26]$ and are rewritten here as

$$
\begin{aligned}
& Z_{m n} \\
& \quad=\left\{\begin{array}{cc}
30\left[0.5772+\ln (2 \kappa l)-C_{i}(2 \kappa l)\right]+j\left[30 S_{i}(2 \kappa l)\right], & m=n, \\
30\left[2 C_{i}\left(u_{0}\right)-C_{i}\left(u_{1}\right)-C_{i}\left(u_{2}\right)\right] & \\
-j\left[30\left(2 S_{i}\left(u_{0}\right)-S_{i}\left(u_{1}\right)-S_{i}\left(u_{2}\right)\right)\right], & m \neq n,
\end{array}\right.
\end{aligned}
$$

where $\kappa$ is the wave number equal to $2 \pi / \lambda$,

$$
\begin{gathered}
u_{0}=\kappa d_{h}, \\
u_{1}=\kappa\left(\sqrt{d_{h}{ }^{2}+l^{2}}+l\right), \\
u_{2}=\kappa\left(\sqrt{d_{h}{ }^{2}+l^{2}}-l\right),
\end{gathered}
$$

$d_{h}$ is the horizontal distance between the two dipole antenna elements. $\mathrm{C}_{i}(\mathrm{u})$ and $\mathrm{S}_{i}(\mathrm{u})$ are the cosine and sine integrals, respectively. They are given as,

$$
\begin{aligned}
& C_{i}(u)=\int_{\infty}^{u}\left(\frac{\cos (x)}{x}\right) d x, \\
& S_{i}(u)=\int_{0}^{\infty}\left(\frac{\sin (x)}{x}\right) d x .
\end{aligned}
$$

Expressions (33) provide quite accurate values for $\left\{Z_{m n}\right\}$ for the dipole spacing of not less than $0.15 \lambda$. If one wishes to have a more accurate model for the mutual coupling effect, the theory and expressions given in [27] are recommended.

Under the presence of mutual coupling, the channels matrix $H$ appearing in expressions (23) and (24) has to be replaced by the new channel matrix $H^{\prime}=C_{R} H C_{T}$ which takes into account the mutual coupling. By taking into account the mutual coupling, the expression for the channel matrix (24) is modified to

$$
H_{\mathrm{mc}}=C_{R} R_{R}^{1 / 2} G_{H} R_{T}^{1 / 2} C_{T} .
$$

The new transmit and receiving correlation matrices can be obtained by introducing,

$$
\begin{aligned}
& R_{\mathrm{Rmu}}=C_{R} R_{R}^{1 / 2}, \\
& R_{\mathrm{Tmu}}=R_{T}^{1 / 2} C_{T} .
\end{aligned}
$$

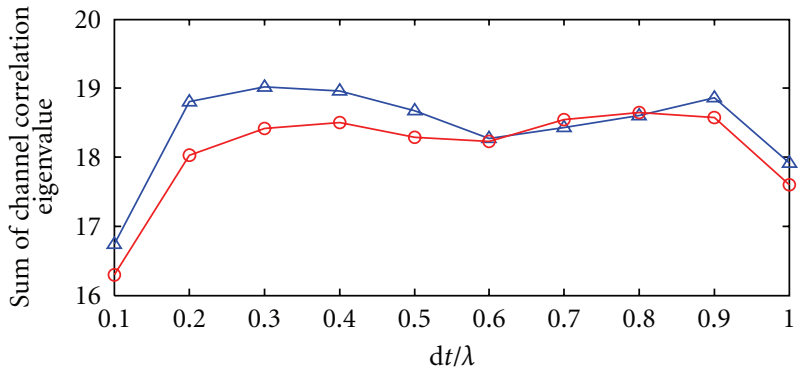

(a) Sum of Channel Correlation Eigenvalues versus Transmitter Antenna Spacing

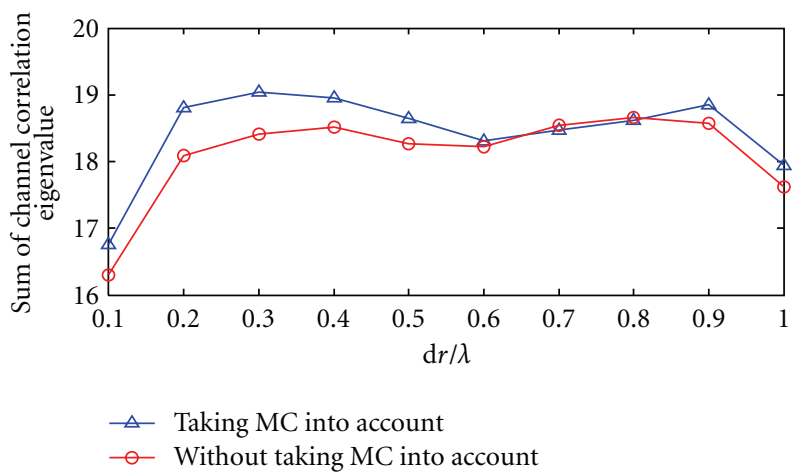

(b) Sum of Channel Correlation Eigenvalues versus Receiver Antenna Spacing

FIGURE 1: Mutual coupling effects on the sum of channel correlation matrix eigenvalues. (a) Sum of eigenvalues versus transmitter antenna element spacing; (b) Sum of eigenvalues versus receiver antenna element spacing.

By using (23) and (24) they can be rewritten as (39) and (38), respectively

$$
\begin{gathered}
H_{\mathrm{mc}}=R_{R \mathrm{mu}} G_{H} R_{T \mathrm{mc}}, \\
R_{H}^{\mathrm{mc}}=H_{\mathrm{mc}}^{H} H_{\mathrm{mc}}=Q_{\mathrm{mc}} \Lambda_{\mathrm{mc}} Q_{\mathrm{mc}}^{H} .
\end{gathered}
$$

Under the mutual coupling conditions, (22) is changed to

$$
\mathrm{MSE}_{\mathrm{MMSE}}^{\mathrm{mc}}=E\left\{\operatorname{tr}\left\{\left(\Lambda_{\mathrm{mc}}^{-1}+\sigma_{n}^{-2} M_{r}^{-1} Q_{\mathrm{mc}}^{H} P P^{H} Q_{\mathrm{mc}}\right)^{-1}\right\}\right\} .
$$

The inclusion of mutual coupling affects the matrix $\Lambda$. Therefore, the sum of the eigenvalues of channel correlation matrix is changed accordingly. Figure 1 shows the simulation results illustrating the effect of mutual coupling on the sum of the eigenvalues. For this simulation, a uniform linear array with 4 antenna elements is assumed at both transmitter and receiver sides. The elements are wire dipoles having length of $0.5 \lambda$. The following normalization of channel matrix $H$ is applied $\|H\|_{F}^{2}=M_{r} M_{t}$, where $\|\cdot\|_{F}^{2}$ is the Frobenius norm.

In Figure 1(a), the antenna element spacing at the receiver is fixed to $1 \lambda$ while the spacing at the transmitter varies from $0.1 \lambda$ to $1 \lambda$. In Figure $1(\mathrm{~b})$, the spacing at the transmitter is fixed to $1 . \lambda$ while the spacing at the receiver varies from $0.1 \lambda$ to $1 \lambda$. From both Figures $1(\mathrm{a})$ and $1(\mathrm{~b})$, one 
can see that when the antenna elements spacing is between $0.1 \lambda$ to $0.5 \lambda$, there is an apparent difference between the sum of channel correlation eigenvalues with and without taking mutual coupling effects into account. The ones with mutual coupling effects are higher than the ones without. When the spacing is increased and exceeds $0.5 \lambda$, the difference becomes smaller and the blue and red curves overlap. As a result, at the antenna spacing between $0.1 \lambda$ and $0.5 \lambda$, the mutual coupling increases the sum of eigenvalues of the channel correlation matrix. This is because within this spacing range the mutual coupling decreases the spatial correlation level. Therefore the channel estimation accuracy is adversely affected.

\section{MMSE Method Performance Optimization Under Mutual Coupling Conditions}

In order to optimize the performance of MMSE trainingbased estimation method, we have to find the optimal training matrix. This optimization problem can be expressed as,

$$
\begin{gathered}
\mathrm{MSE}_{\mathrm{MMSE}}^{\mathrm{mc}}=\min _{\|P\|_{F}^{2}=P} \operatorname{tr}\left\{\left(\Lambda_{\mathrm{mc}}^{-1}+\sigma_{n}^{-2} M_{r}^{-1} Q_{\mathrm{mc}}^{H} P P^{H} Q_{\mathrm{mc}}\right)^{-1}\right\}, \\
P^{\mathrm{opt}}=\underset{p}{\arg \min \operatorname{tr}}\left\{\left(\Lambda_{\mathrm{mc}}^{-1}+\sigma_{n}^{-2} M_{r}^{-1} Q_{\mathrm{mc}}^{H} P P^{H} Q_{\mathrm{mc}}\right)^{-1}\right\}, \\
\text { subject to }\|P\|_{F}^{2}=P .
\end{gathered}
$$

By denoting the combined training matrix as

$$
\bar{P}=\sigma_{n}^{-1} M_{r}^{-1 / 2} Q_{\mathrm{mc}}^{H} P,
$$

expression (41) can be rewritten as

$$
\mathrm{MSE}_{\mathrm{MMSE}}^{\mathrm{mc}}=\min _{\|P\|_{F}^{2}=P} \operatorname{tr}\left\{\left(\Lambda_{\mathrm{mc}}^{-1}+\bar{P} \bar{P}^{H}\right)^{-1}\right\} .
$$

Based on properties of the unitary eigenvector matrix $Q_{\mathrm{mc}}$, $\bar{P} \bar{P}^{H}$ has a diagonal structure as given by

$$
\bar{P} \bar{P}^{H}=\left[\begin{array}{llll}
p_{1}^{2} & & & \\
& p_{2}^{2} & & \\
& & \ddots & \\
& & & p_{M_{t}}^{2}
\end{array}\right],
$$

where $p_{i}^{2}$ is the power of each combined training symbol.

When no optimization is applied, the transmit power is equally distributed into the training symbols as given by

$$
p_{i}^{2}=\sigma_{n}^{-2} M_{r}^{-1} M_{t}^{-1} P .
$$

Next (44) can be rewritten as,

$$
\begin{array}{r}
\operatorname{MSE}_{\mathrm{MMSE}}^{\mathrm{mc}}=\min _{\|P\|_{F}^{2}=P} \sum_{i=1}^{M_{t}}\left(\lambda_{i, \mathrm{mc}}^{-1}+p_{i}^{2}\right)^{-1} \\
\text { assume } M_{t}=M_{r},
\end{array}
$$

in which $\lambda_{i, \mathrm{mc}}$ is the $i$ th eigenvalue in $\Lambda$.

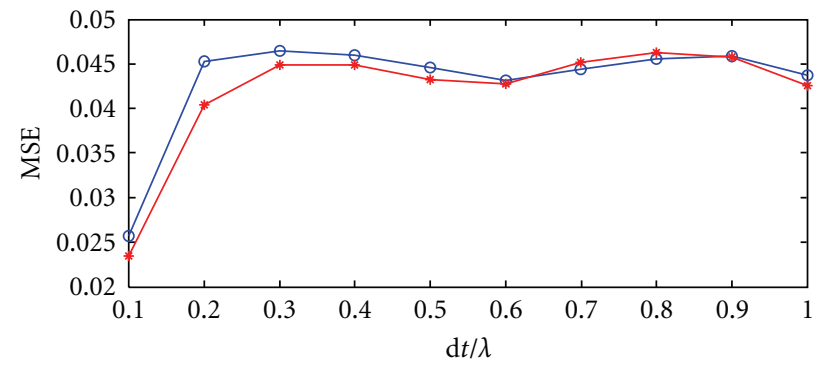

(a) MMSE MSE versus Transmitter Antenna Spacing

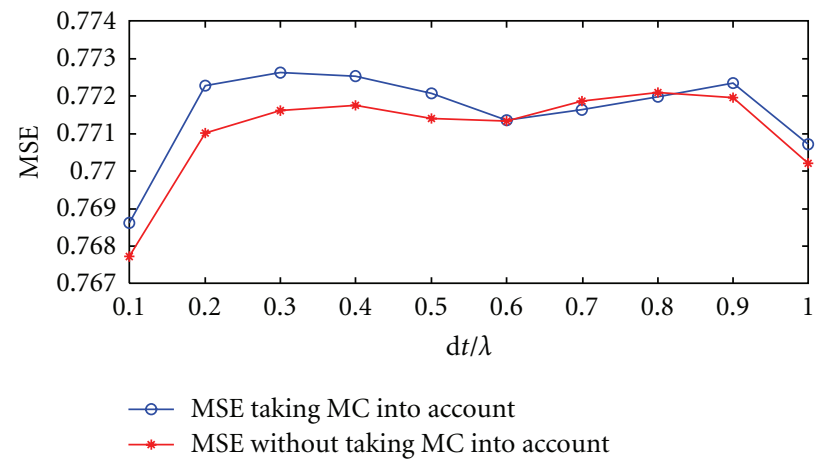

(b) SLS MSE versus Transmitter Antenna Spacing

FIGURE 2: MMSE and SLS MSE performance versus transmitter antenna element spacing.

To minimize MSE in (44), a water-filling based algorithm can be applied to optimize the power allocation to training symbols. The symbols in the optimal training matrix are given as $[9,10]$

$$
p_{i}=\sqrt{\left(\mu-\lambda_{i, \mathrm{mc}}^{-1}\right)^{+}}
$$

in which $(x)^{+}=\max (x, 0)$ and $\mu$ needs to be found to satisfy $\|P\|_{F}^{2}=P$.

Finally, the optimal training matrix is given as

$$
P^{\mathrm{opt}}=\sigma_{n}^{-2} M_{r}^{-1} Q_{\mathrm{mc}}\left(\left[\mu I-\Lambda_{\mathrm{mc}}^{-1}\right]^{+}\right)^{1 / 2} .
$$

\section{Simulation Results}

This section reports the computer simulations aiming at the assessment of effects of mutual coupling on the trainingbased MIMO channel estimation methods. In the undertaken investigation, the transmitter and receiver are assumed to be equipped with 4-element uniform array antennas. The elements are wire dipoles having length of $0.5 \lambda$. The antenna element spacing at the receiver is fixed to $1 \lambda$ while the spacing at the transmitter varies from $0.1 \lambda$ to $1 \lambda$. Equations (38) and (24) are used to construct the MIMO channel with and without taking mutual coupling effects into account. The transmit SPNR is fixed at $25 \mathrm{~dB}$.

Figure 2 presents the MSE performance of MMSE and SLS methods versus transmitter antenna elements spacing. In this figure, the blue and red curves indicate with and without taking into account mutual coupling cases, respectively. For 


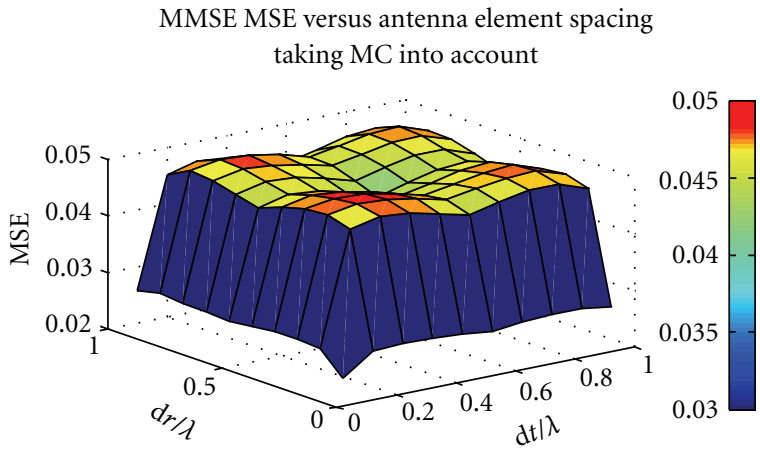

(a) 3D view

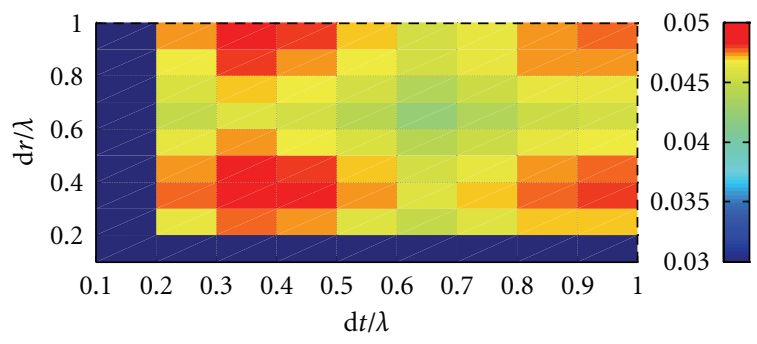

(b) $2 \mathrm{D}$ view

FIGURE 3: MMSE MSE versus transmitter and receiver antenna element spacing taking MC into account.

both MMSE and SLS methods, an apparent difference occurs between the MSE performance with and without taking mutual coupling into account when the transmitter antenna element spacing varies from $0.1 \lambda$ to $0.5 \lambda$. The MSE obtained for taking into account mutual coupling effects is worse than the MSE obtained without taking into account the mutual coupling. When the antenna element spacing is larger than $0.5 \lambda$, the difference disappears. This finding agrees with the prediction based on the sum of channel correlation eigenvalues that shows that for the spacing between $0.1 \lambda$ to $0.5 \lambda$ the mutual coupling undermines the estimation accuracy.

Further investigations are done by varying the antenna element spacing in both transmitter and receiver arrays. The results are given in Figures 3, 4, 5 and 6. In each figure, there are two subfigures showing three dimensional (3D) and two dimensional (2D) views. Figure 3 shows the MMSE MSE performance under the impact of mutual coupling. One can see that the worst estimation error occurs when the antenna element spacing in the transmitter and receiver is between $0.3 \lambda$ and $0.4 \lambda$. Figure 3 shows the MMSE MSE performance without taking into account the mutual coupling. When the antenna element spacing in the transmitter and receiver is between $0.5 \lambda$ and $0.7 \lambda$, MSE achieves its best performance. Similar results are also obtained for the SLS method, as presented in Figures 5 and 6.

Figure 7 shows the results for the MMSE estimation method when the training sequence is optimized. For comparison purposes, the results for nonoptimized MMSE are also plotted. One can see that with the optimized training symbols, the channel estimation error decreases. However,
MSE versus antenna element spacing without taking MC into account

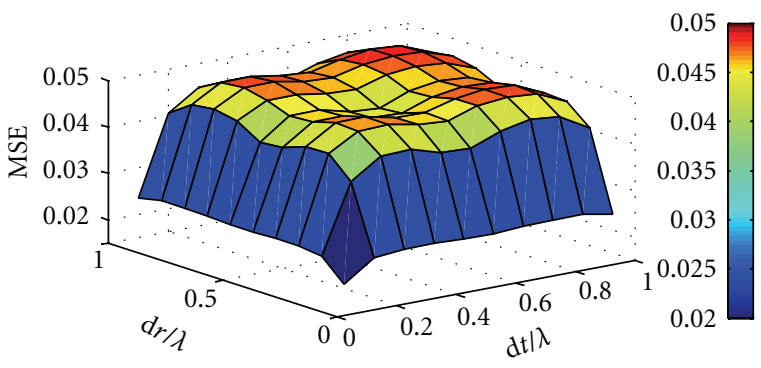

(a) $3 \mathrm{D}$ view

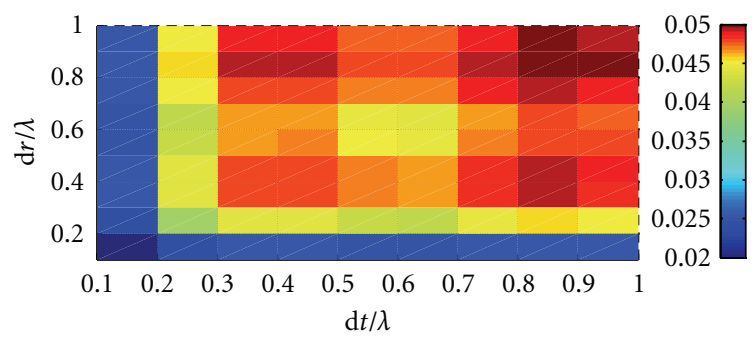

(b) $2 \mathrm{D}$ view

Figure 4: MMSE MSE versus transmitter and receiver antenna element spacing without taking MC into account.

the optimization does not narrow the difference between the two sets of results with and without taking mutual coupling into account when the antenna element spacing is between $0.1 \lambda$ to $1.0 \lambda$. In the case of a compact multiple antenna mobile communication device whose antenna element spacing is between $0.1 \lambda$ to $0.5 \lambda$, the antenna decoupling is a required to achieve a better channel estimation performance.

Figure 8 further demonstrates the advantages of the optimization method. For the undertaken simulation, the antenna element spacing at the receiver is fixed to $1 \lambda$ while the spacing at the transmitter is made $0.2 \lambda$. From the presented results one can see that the performances of MSE with optimization are always better than the ones without it. At a lower transmit SPNR, the results for MSE with optimization are significantly improved. The results also show that when the transmitter antenna element spacing is within $0.1 \lambda$ to $0.5 \lambda$, the MSE taking into account mutual coupling is worse than the one without taking into account mutual coupling. This is irrespective from whether optimization is used or not.

\section{Conclusion}

This paper has reported the investigations on the trainingbased channel estimation methods for a narrowband MIMO system, in which both the transmitter and the receiver are equipped with multiple element antennas in the form of wire dipoles. The investigations have included the effect of mutual coupling in addition to spatial correlation that is present at the transmitting and receiving array antennas due to surrounding scattering objects. The spatial correlation has been taken into account using the Kronecker model, in 
SLS MSE versus antenna element spacing taking $\mathrm{MC}$ into account

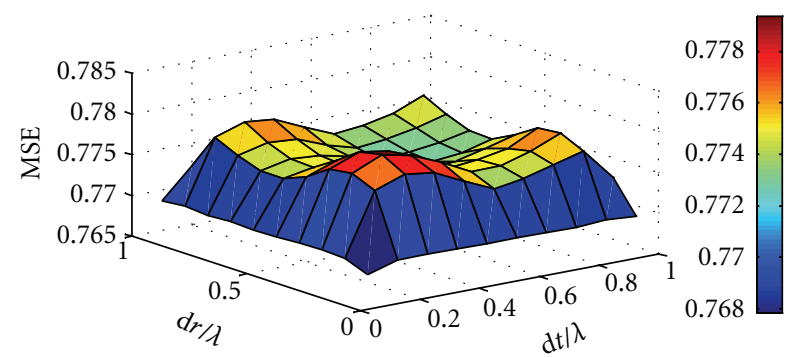

(a) $3 \mathrm{D}$ view

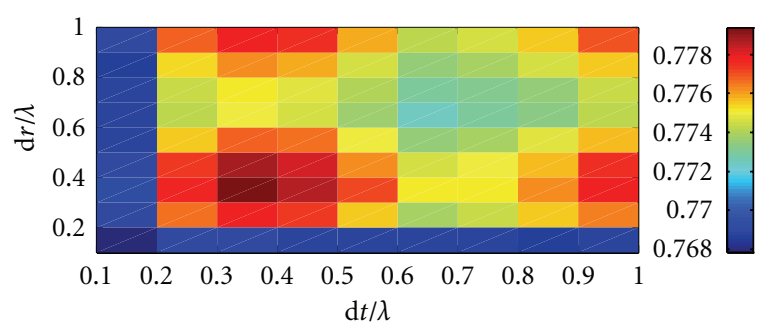

(b) $2 \mathrm{D}$ view

FigURE 5: SLS MSE versus transmitter and receiver antenna element spacing taking MC into account.

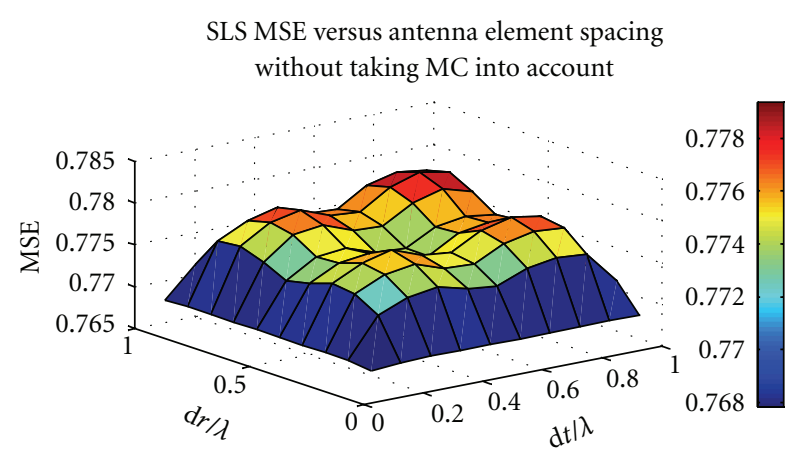

(a) $3 \mathrm{D}$ view

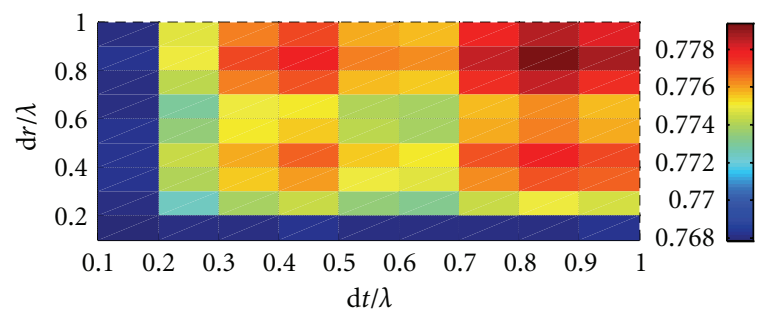

(b) $2 \mathrm{D}$ view

FIGURE 6: SLS MSE versus transmitter and receiver antenna element spacing without taking MC into account.

which correlation matrices for the transmitting and receiving sides are separated. The mutual coupling has been included using the closed-form expressions for impedance matrices of parallel side-to-side wire dipoles. Two cases of nonoptimized and optimized training sequence transmission schemes have been considered. The computer simulations have been

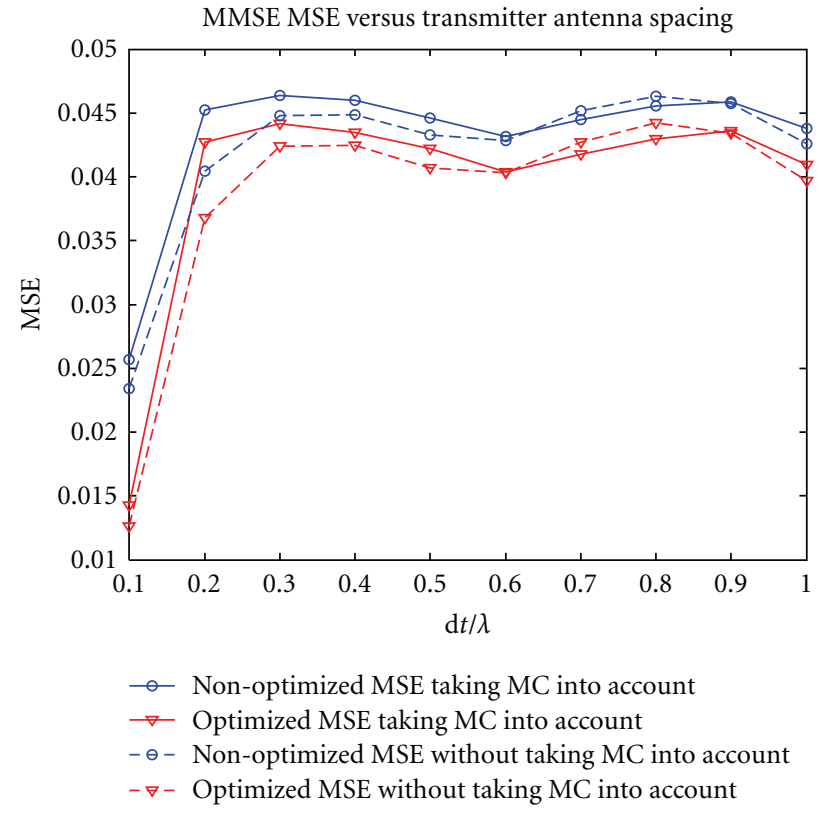

FIGURE 7: Optimized and nonoptimized MMSE MSE versus transmitter antenna element spacing with and without taking mutual coupling effects into account.

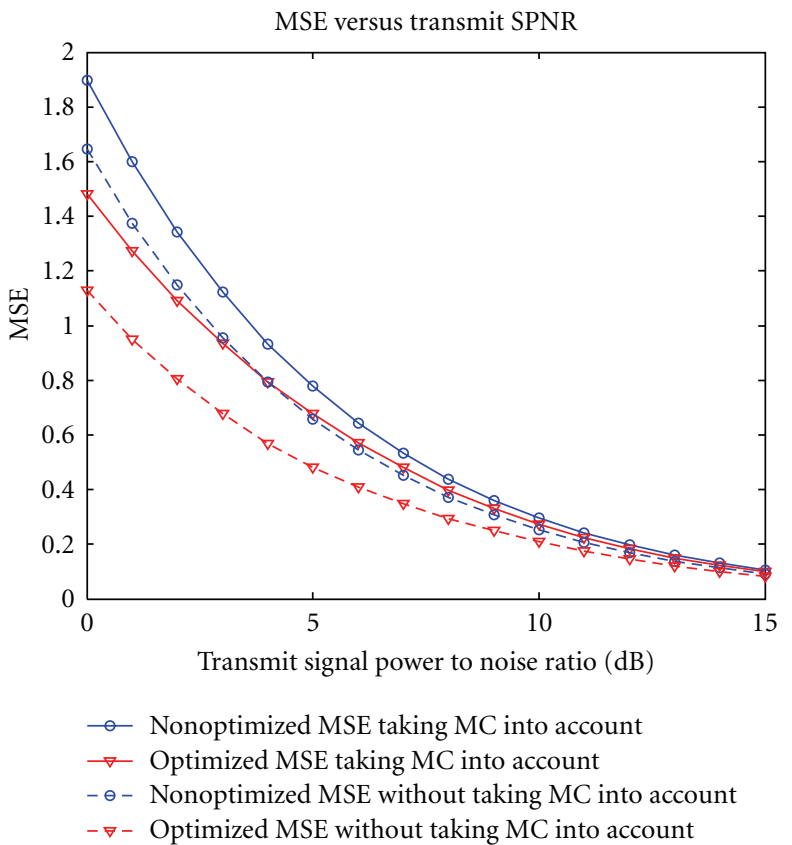

FIGURE 8: MMSE MSE for versus transmit SPNR with and without taking mutual coupling effects into account for optimized and nonoptimized cases.

carried out for the case when the transmitting and receiving sides are equipped with four-element uniform half-wave dipole arrays surrounded by circles of uniformly distributed scattering objects. The obtained simulation results have shown that at the antenna (dipole) spacing between $0.1 \lambda$ to $0.5 \lambda$, mutual coupling decreases the spatial correlation and 
adversely affects the channel estimation offered by MMSE and SLS methods. When the spacing is larger than $0.5 \lambda$ the MSE is slightly different when the mutual coupling is taken into account or neglected. Next considerations have focused on optimization of training signals for MIMO channel estimation. When the optimization of the training sequence is applied, an improvement in MSE has been demonstrated irrespectively whether the mutual coupling effects are neglected or taken into account. This improvement is more significant at a lower transmit SPNR. The difference in MSE for with and without taking into account mutual coupling effects is unchanged when the antenna element spacing is within $0.1 \lambda$ to $0.5 \lambda$. This means that for a compact multiple antenna mobile communication device the improvement of MSE requires decoupling of the antenna elements to achieve a better estimation of MIMO channel.

\section{References}

[1] E. Telatar, "Capacity of multi-antenna Gaussian channels," European Transactions on Telecommunications, vol. 10, no. 6, pp. 585-595, 1999.

[2] T. L. Marzetta and B. M. Hochwald, "Capacity of a mobile multiple-antenna communication link in Rayleigh flat fading," IEEE Transactions on Information Theory, vol. 45, no. 1, pp. 139-157, 1999.

[3] A. Narula, M. J. Lopez, M. D. Trott, and G. W. Wornell, "Efficient use of side information in multiple-antenna data transmission over fading channels," IEEE Journal on Selected Areas in Communications, vol. 16, no. 8, pp. 1423-1436, 1998.

[4] C. Budianu and L. Tong, "Channel estimation for spacetime orthogonal block codes," IEEE Transactions on Signal Processing, vol. 50, no. 10, pp. 2515-2528, 2002.

[5] A. Grant, "Joint decoding and channel estimation for linear MIMO channels," in Proceedings of the IEEE Wireless Communications and Networking Conference, pp. 1009-1012, Chicago, Ill, USA, September 2000.

[6] A. S. Kyung, R. W. Heath, and B. K. Heung, "Shannon capacity and symbol error rate of space-time block codes in MIMO Rayleigh channels with channel estimation error," IEEE Transactions on Wireless Communications, vol. 7, no. 1, pp. 324-333, 2008.

[7] X. Zhang and B. Ottersten, "Performance analysis of V-BLAST structure with channel estimation errors," in Proceedings of the 4th IEEE Workshop on Signal Processing Advances in Wireless Communications (SPAWC '03), pp. 487-491, Rome, Italy, June 2003.

[8] P. Layec, P. Piantanida, R. Visoz, and A. O. Berthet, "Capacity bounds for MIMO multiple access channel with imperfect channel state information," in Proceedings of the IEEE Information Theory Workshop (ITW'08), pp. 21-25, Porto, Portugal, May 2008.

[9] M. Biguesh and A. B. Gershman, "MIMO channel estimation: optimal training and tradeoffs between estimation techniques," in Proceedings of the IEEE International Conference on Communications (ICC '04), vol. 5, pp. 2658-2662, Paris, France, June 2004.

[10] M. Biguesh and A. B. Gershman, "Training-based MIMO channel estimation: a study of estimator tradeoffs and optimal training signals," IEEE Transactions on Signal Processing, vol. 54, no. 3, pp. 884-893, 2006.
[11] T. Svantesson and A. Ranheim, "Mutual coupling effects on the capacity of multi-element antenna systems," in Proceedings of the IEEE International Conference on Acoustics, Speech, and Signal Processing (ICASSP '01), pp. 2485-2488, Las Vegas, Nev, USA, April 2001.

[12] J. W. Wallace and M. A. Jensen, "The capacity of MIMO wireless systems with mutual coupling," in Proceedings of the IEEE Vehicular Technology Conference (VTC '02), pp. 696-700, Vancouver, Canada, 2002.

[13] J. W. Wallace and M. A. Jensen, "Mutual coupling in MIMO wireless systems: a rigorous network theory analysis," IEEE Transactions on Wireless Communications, vol. 3, no. 4, pp. 1317-1325, 2004.

[14] P. N. Fletcher, M. Dean, and A. R. Nix, "Mutual coupling in multi-element array antennas and its influence on MIMO channel capacity," Electronics Letters, vol. 39, no. 4, pp. 342344, 2003.

[15] S. Lu, H. T. Hui, M. Bialkowski, and X. Liu, "Optimizing transmission strategy in single-user MIMO systems under the influence of antenna mutual coupling," ECTI Transactions on Electrical Engineering Electronics, and Communications, vol. 6, no. 2, pp. 73-78, 2008.

[16] S. Lu, X. Liu, H. T. Hui, M. Bialkowski, N. Seman, and H. Y. Zhang, "Power allocation strategy for compact MIMO transmitters with Uniform Circular Arrays under the influence of antenna mutual coupling," in Proceedings of the IEEE Asia Pacific Microwave Conference (APMC '07), pp. 11-14, Bangkok, Thailand, December 2007.

[17] S. Lu, H. T. Hui, M. Bialkowski, and X. Liu, "Optimizing transmission strategy in single-user MIMO systems under the influence of antenna mutual coupling," in Proceedings of the IEEE International Symposium on Communications and Information Technologies (ISCIT '07), pp. 370-374, Sydney, Australia, October 2007.

[18] M. E. Bialkowski, P. Uthansakul, K. Bialkowski, and S. Durrani, "Investigating the performance of MIMO systems from an electromagnetic perspective," Microwave and Optical Technology Letters, vol. 48, no. 7, pp. 1233-1238, 2006.

[19] X. Liu, S. Lu, M. E. Bialkowski, and H. T. Hui, "MMSE channel estimation for MIMO system with receiver equipped with a circular array antenna," in Proceedings of the AsiaPacific Microwave Conference (APMC'07), Bangkok, Thailand, December 2007.

[20] S. Lu, H. T. Hui, M. E. Bialkowski, X. Liu, H. S. Lui, and N. V. Shuley, "The effect of antenna mutual coupling on channel estimation of MIMO-OFDM systems," in Proceedings of the IEEE Antennas and Propagation Society International Symposium (APS '07), pp. 2945-2948, Honolulu, Hawaii, USA, June 2007.

[21] S. M. Kay, Fundamentals of Statistical Signal Processing: Estimation Theory, Prentice-Hall, Upper Saddle River, NJ, USA, 1993.

[22] E. G. Larsson and P. Stoica, Space-Time Block Coding for Wireless Communication, Cambridge University Press, Cambridge, UK, 2003.

[23] C.-N. Chuah, D. N. C. Tse, J. M. Kahn, and R. A. Valenzuela, "Capacity scaling in MIMO wireless systems under correlated fading," IEEE Transactions on Information Theory, vol. 48, no. 3, pp. 637-650, 2002.

[24] S. Da-Shan, G. J. Foschini, M. J. Gans, et al., "Fading correlation and its effect on the capacity of multi-element antenna systems," in Proceedings of the IEEE International Conference on Universal Personal Communications (ICUPC'98), Florence, Italy, 1998. 
[25] X. Liu, M. E. Bialkowski, and F. Wang, "Investigations into the effect of spatial correlation on channel estimation and capacity of multiple input multiple output system," International Journal of Communications, Network and System Sciences, vol. 2, no. 3, 2009.

[26] S. Durrani and M. E. Bialkowski, "Effect of mutual coupling on the interference rejection capabilities of linear and circular arrays in CDMA systems," IEEE Transactions on Antennas and Propagation, vol. 52, no. 4, pp. 1130-1134, 2004.

[27] H. T. Hui, "Improved compensation for the mutual coupling effect in a dipole array for direction finding," IEEE Transactions on Antennas and Propagation, vol. 51, no. 9, pp. 2498-2503, 2003. 

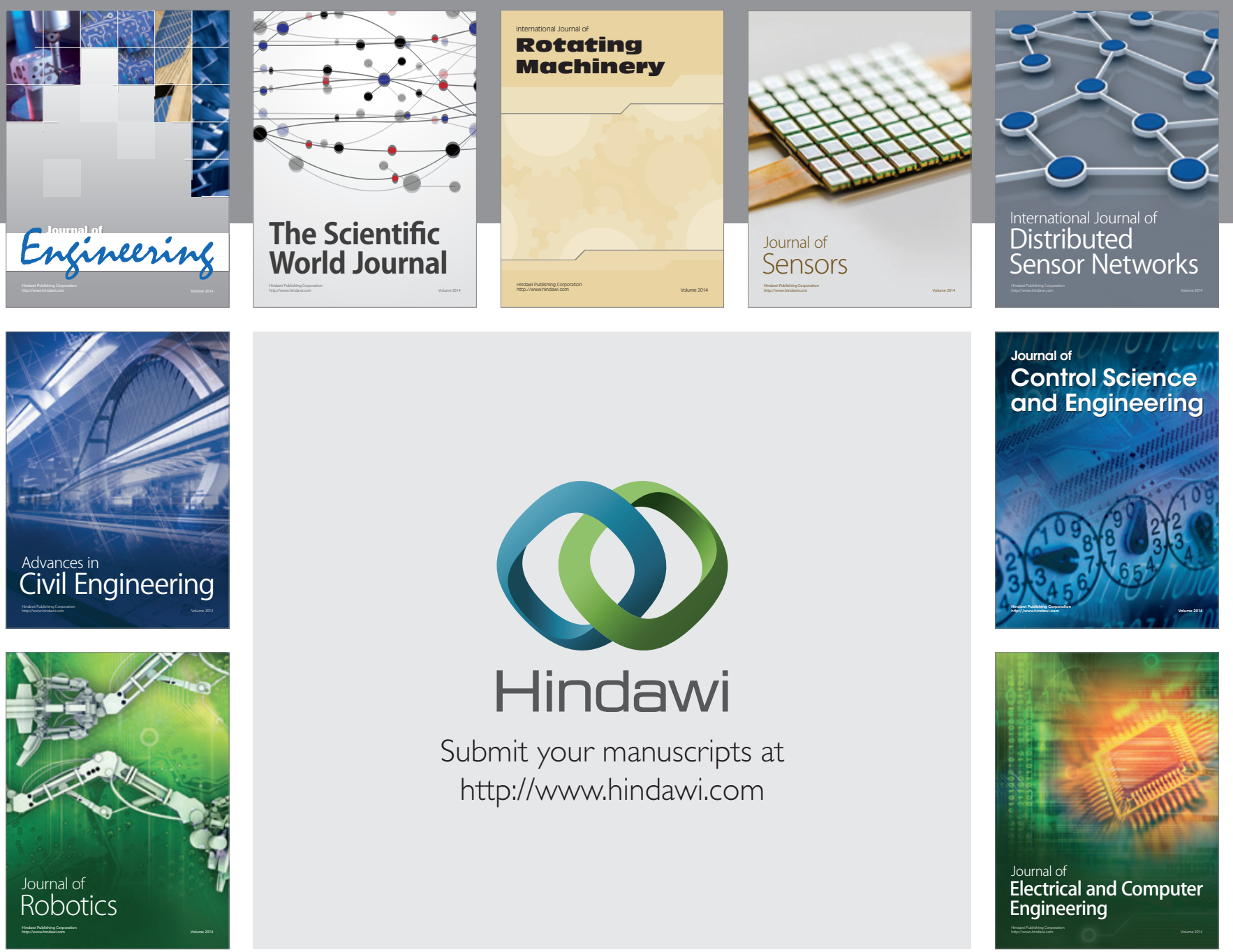

Submit your manuscripts at

http://www.hindawi.com
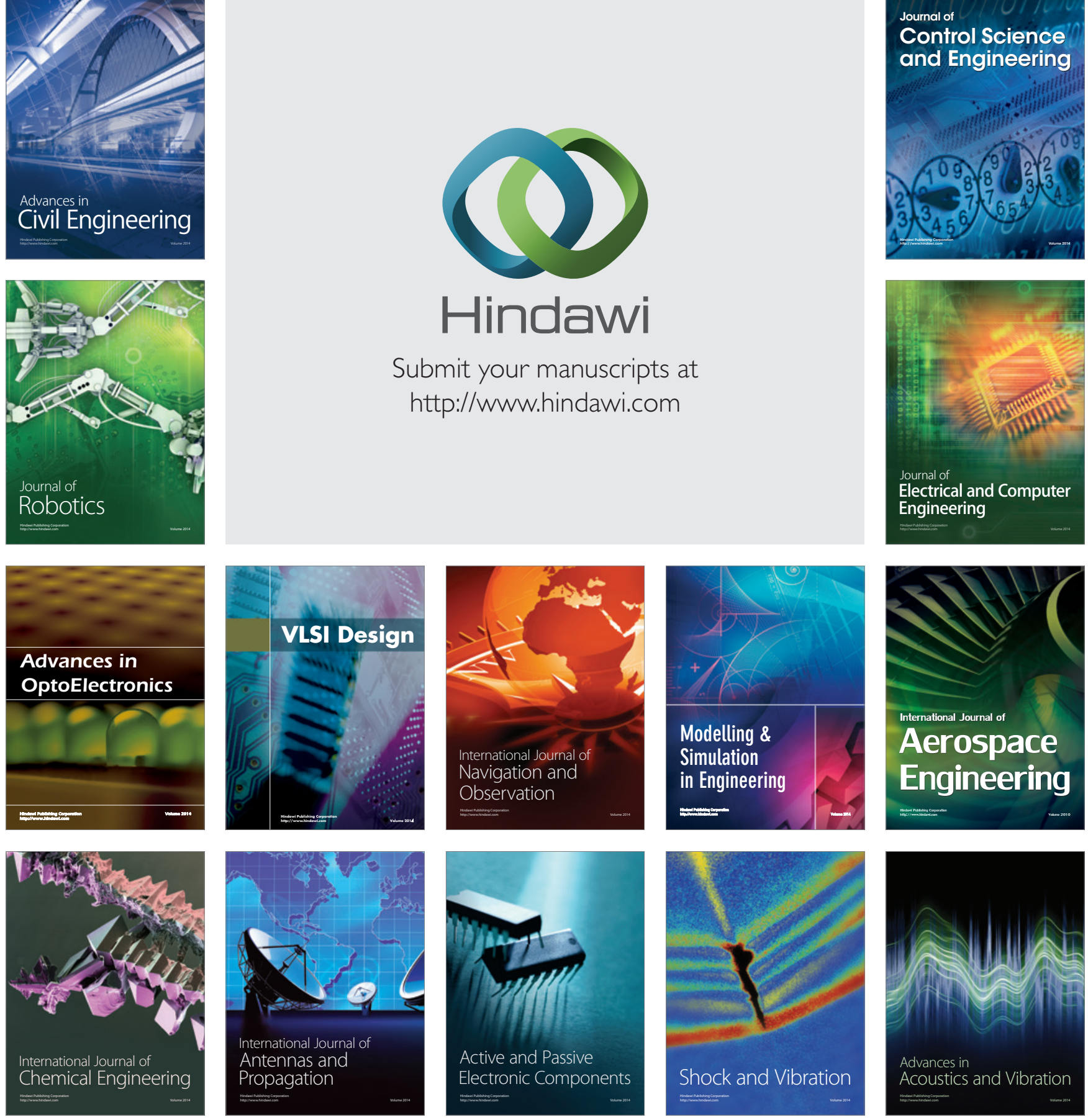